\title{
Non-Alcoholic Fatty Liver Disease as a Coronary Heart Disease Severity Predictor
}

\author{
Hossam Ismael1, Mohammed Tag-Adeen 1* , Ahmed Abdel-Rady², Mohamed Shazly ${ }^{3}$, \\ Ahmed Hussein 4
}

\author{
${ }^{1}$ Department of Internal Medicine, Faculty of Medicine, South Valley University, Qena, Egypt \\ ${ }^{2}$ Department of Radio-Diagnosis, Al-Azhar University, Assiut, Egypt \\ ${ }^{3}$ Department of Radio-Diagnosis, Al-Azhar University, Cairo, Egypt \\ ${ }^{4}$ Department of Internal Medicine, Faculty of Medicine, Sohag University, Sohag, Egypt \\ Email: dr_hossam_ismail@yahoo.com, *tagmedicine@gmail.com,dr_ahmedazkool@yahoo.com, \\ mohamadshazly5@yahoo.com,forahmedaly@yahoo.com
}

How to cite this paper: Ismael, $\mathrm{H}$., Tag-Adeen, M., Abdel-Rady, A., Shazly, M. and Hussein, A. (2020) Non-Alcoholic Fatty Liver Disease as a Coronary Heart Disease Severity Predictor. International Journal of Clinical Medicine, 11, 182-192.

https://doi.org/10.4236/ijcm.2020.114018

Received: March 19, 2020

Accepted: April 14, 2020

Published: April 17, 2020

Copyright $\odot 2020$ by author(s) and Scientific Research Publishing Inc. This work is licensed under the Creative Commons Attribution International License (CC BY 4.0).

http://creativecommons.org/licenses/by/4.0/

\begin{abstract}
Background and Aim: Association of non-alcoholic fatty liver disease (NAFLD) and liver related mortality is well-known; however; its cardiovascular risk remains questionable. The aim of this work was to highlight the association between NAFLD and the severity of coronary heart disease. Method: The study was conducted between July and December 2019 at Qena university hospital, Qena, Egypt on patients who were scheduled for coronary angiography. Liver steatosis was diagnosed using FibroScan and the severity of coronary artery occlusion was assessed by Gensini score calculation. Statistical analysis was done to study the correlation between liver steatosis and angiography findings. Result: A total of 100 patients were included, mean age 48 years $( \pm 8 \mathrm{SD})$. Correlation between coronary angiography and the baseline variables revealed statistically significant positive correlation between Gensini score and ALT ( $\mathrm{p}=0.002)$, FBS $(\mathrm{p}<0.001)$, steatosis $(\mathrm{p}<0.001)$ and diabetes $(\mathrm{p}<0.001)$. Also, gradual increase in the Gensini score with higher steatosis grades was noticed, medians: 10.5, 21.7, 57.6 and 102.6 in S0, S1, S2 and S3 respectively, $\mathrm{p}=0.01$. Variables that have significantly predicted higher Gensini score in univariable regression analysis were: diabetes (OR: 55.6, CI: (41 70)), hypertension (OR: 19, CI: (2 - 37)), F2-F4 fibrosis (OR: 33, CI: (24 - 38)) and S2-S3 steatosis (OR: 34, (23 - 40)), while in multivariable regression; the statistically significant variables were: diabetes (OR: 23, CI: (10 - 37)), hypertension (OR: 11, CI: (4.8 - 22)) and S2-S3 steatosis (OR: 24, CI: (17 - 31)). Conclusion: NAFLD is an independent cardiovascular risk predictor with statistically significant increase in Gensini score with the higher grades of NAFLD.
\end{abstract}


Keywords

NAFLD, FibroScan, Gensini Score

\section{Introduction}

Non-alcoholic fatty liver disease (NAFLD) represents a worldwide health problem with an increasing prevalence reaching about $75 \%$ of chronic liver disease (CLD) in the developed countries, which makes it the most common CLD in the western world [1]. In a recent meta-analysis, the global prevalence of NAFLD was estimated to be around 25\% with highest prevalence in the Middle East and South America and lowest in Africa [2].

Traditionally, NAFLD has linked to liver-related morbidities through its progression to non-alcoholic steatohepatitis (NASH) with subsequent liver cirrhosis, and it is expected that NAFLD related liver diseases will be the leading cause of liver transplantation by 2030 [3]. In addition to this obvious link between NAFLD and end-stage liver disease, several studies suggested the presence of an association between NAFLD and cardiovascular disease (CVD). Recently, a large body of evidence has supported this suggestion and classified CVD as the main cause of death in patients with NAFLD [4] and considered NAFLD as a significant independent risk factor for subclinical and clinical CVD in the absence of the classic cardiovascular risk factors [5] [6].

In contrary to the traditional cardiovascular risk factors like diabetes and obesity, and despite the growing evidence for the association of CVD with NAFLD, the exact mechanism of this association and the degree of correlation between the grade of NAFLD and the severity of CVD are not well-studied [7] [8] [9]. The aim of our work was to study the correlation between NAFLD and the severity of coronary heart disease (CHD) as determined by Gensini score.

\section{Materials and Method}

\subsection{Patients}

Patients without prior history of definite ischemic event who were scheduled for coronary angiography from July 2019 - December 2019 at Qena university hospital, Qena, Egypt were included, while those with known history of definite CVD or heart failure were initially excluded. Patients with prior history of CLD including chronic hepatitis $\mathrm{C}$ ( $\mathrm{CHC})$, chronic hepatitis $\mathrm{B}(\mathrm{CHB})$ and NAFLD/NASH or renal impairment were excluded. After initial abdominal ultrasound (US), patients with definite cirrhotic echo pattern or any confounding factor interferes with accurate transient elastography (TE) reading such as hepatocellular carcinoma, ascites or morbid obesity were excluded.

\subsection{Transient Elastography (TE)}

Liver stiffness measurement (LSM) and controlled attenuation parameter (CAP) 
were obtained by an expert operator using FibroScan ${ }^{\bullet}$ device (Echosense, Paris, France). The procedure was performed after 8-hour fasting while the patient in the supine position. Result was not considered reliable except after acquisition of 12 successful readings with interquartile range/median ratio less than $30 \%$ [10]. LSM was used to estimate the METAVIR fibrosis stage as follows: F0-F1: 2.5 $6.9 \mathrm{kPa}$; F2: 7.0 - $9.4 \mathrm{kPa}$; F3: 9.5 - $12.4 \mathrm{kPa}$; F4: $\geq 12.5 \mathrm{kPa}$ [11]. CAP was expressed in $\mathrm{dB} / \mathrm{m}$ and its values were used to estimate steatosis stage as follows: $\mathrm{S} 0$ $<238 \mathrm{~dB} / \mathrm{m}, \mathrm{S} 1: 238-258 \mathrm{~dB} / \mathrm{m}, \mathrm{S} 2: 259-291 \mathrm{~dB} / \mathrm{m}$ and S3: $\geq 292 \mathrm{~dB} / \mathrm{m}$ [12].

\subsection{Coronary Angiography}

Coronary angiography was performed within 4 weeks of enrollment by an expert cardiologist blinded about the LSM \& CAP readings of the patients. Gensini score was then calculated as mentioned in the literature [13] [14].

\subsection{The Studied Variables}

Gensini score will be correlated to the continuous variables including: age, fasting blood sugar (FBS), total serum cholesterol (TC), triglycerides, high density lipoprotein (HDL), alanine transaminase (ALT), aspartate transaminase (AST) and serum bilirubin, and categorical variables including: gender, diabetes mellitus (DM), hypertension (HTN), smoking, liver fibrosis (F0-F4) and liver steatosis (S0-S3).

\subsection{Statistical Analysis}

Categorical variables are expressed as number and percent, continuous variables as median and interquartile ranges (IQR). Chi squared test was used to compare non-parametric variables. Pearson correlation was used to study the correlation between Gensini score and other variables. Univariate and multivariate logistic regression was calculated to identify the statistically significant CVD risk predictors. Analysis was performed using SPSS 'version 22. $\mathrm{p}<0.05$ was considered statistically significant.

\subsection{Ethical Clearance}

The study protocol was concomitant with the ethical guidelines of the 1975 Declaration of Helsinki and approved by the ethical committee of Qena Faculty of Medicine, South Valley University, Qena, Egypt. A written informed consent was obtained from all included patients before enrollment in this study.

\section{Result}

A total of 104 patients were initially enrolled in this work, 4 patients were excluded from our analysis; 3 had abnormal renal biochemistry and 1 had microalbuminuria. The baseline criteria of the finally included 100 patients are shown in Table 1; the mean age was 48 years $( \pm 8 \mathrm{SD}), 30 \%$ were females, $52 \%$ were smokers, $30 \%$ had type 2 diabetes and $36 \%$ had essential hypertension. The rest 
Table 1. Baseline criteria in the studied patients. Categorical variables are expressed as number and percent while continuous variables are expressed as median and interquartile ranges (IQR 25 \& IQR 75) except the age which is expressed as mean and standard deviation.

\begin{tabular}{|c|c|c|}
\hline \multicolumn{2}{|c|}{ Baseline Variables } & Studied Patients $(n=100)$ \\
\hline \multicolumn{2}{|c|}{ Age (years) } & $47.9 \pm 8.4($ Mean $\pm S D)$ \\
\hline \multicolumn{2}{|c|}{ Females } & $30(30 \%)$ \\
\hline \multicolumn{2}{|c|}{ Smokers } & $52(52 \%)$ \\
\hline \multicolumn{2}{|c|}{ Hypertensive } & $36(36 \%)$ \\
\hline \multicolumn{2}{|c|}{ Diabetics } & $30(30 \%)$ \\
\hline \multicolumn{2}{|c|}{ Fatty Liver in the US } & $42(42 \%)$ \\
\hline & So & $12(12 \%)$ \\
\hline \multirow{5}{*}{ Steatosis } & S1 & $38(38 \%)$ \\
\hline & S2 & $22(22 \%)$ \\
\hline & S3 & $28(28 \%)$ \\
\hline & F0 & $16(16 \%)$ \\
\hline & $\mathrm{F} 1$ & $56(56 \%)$ \\
\hline \multirow[t]{3}{*}{ Fibrosis } & $\mathrm{F} 2$ & $24(24 \%)$ \\
\hline & F3 & $2(2 \%)$ \\
\hline & $\mathrm{F} 4$ & $2(2 \%)$ \\
\hline \multicolumn{2}{|c|}{ RBS (mg/dl) } & $110(100-150)$ \\
\hline \multicolumn{2}{|c|}{ Total Cholesterol (mg/dl) } & $204.5(162-237)$ \\
\hline \multicolumn{2}{|c|}{$\mathrm{HDL}(\mathrm{mg} / \mathrm{dl})$} & $38.5(35-41)$ \\
\hline \multicolumn{2}{|c|}{ Triglycerides (mg/dl) } & $179(105-260)$ \\
\hline \multicolumn{2}{|c|}{$\operatorname{ALT}(\mathrm{U} / \mathrm{L})$} & $30(17-53)$ \\
\hline \multicolumn{2}{|c|}{ AST (U/L) } & $24(17-35)$ \\
\hline \multicolumn{2}{|c|}{ Bilirubin (mg/dl) } & $0.57(0.4-0.86)$ \\
\hline
\end{tabular}

of the baseline criteria including liver fibrosis (F0-F5) \& steatosis (S0-S3), lipid profile, random blood sugar (RBS), serum creatinine, alanine transaminase (ALT), aspartate transaminase (AST), serum bilirubin and serum albumin were also shown.

Correlation between the result of coronary angiography and the continuous baseline variables has revealed statistically significant positive correlation between Gensini score with ALT ( $\mathrm{r}=0.4, \mathrm{p}=0.002)$ and FBS $(\mathrm{r}=0.6, \mathrm{p}<0.001)$, while the rest of variables showed statistically insignificant correlation, Table 2.

Correlation between Gensini score and categorical variables is shown in Table 3 , in which fibrosis, steatosis and diabetes have shown statistically significant positive correlation $(\mathrm{p}<0.001)$. The other categorical variables including hypertension, smoking and female gender had statistically insignificant correlation.

Figure 1 shows a statistically significant difference between the Gensini score in patients with early fibrosis versus those with significant fibrosis (median: 24 versus $74, p=0.002$ ), while Figure 2 shows gradual increment in the Gensini 
Table 2. Correlation between Gensini score and the continuous variables.

\begin{tabular}{ccc}
\hline Variables & $(\mathbf{r})$ & p-value \\
\hline Age (Years) & 0.03 & 0.8 \\
Total Cholesterol (mg/dl) & 0.16 & 0.2 \\
Triglycerides (mg/dl) & 0.19 & 0.1 \\
HDL (mg/dl) & -0.2 & 0.1 \\
ALT (U/L) & 0.43 & 0.002 \\
AST (U/L) & 0.034 & 0.8 \\
Bilirubin (mg/dl) & 0.19 & 0.1 \\
FBS (mg/dl) & 0.65 & $<0.001$ \\
\hline
\end{tabular}

(r): Pearson correlation coefficient.

Table 3. Correlation between Gensini score and the categorical variables.

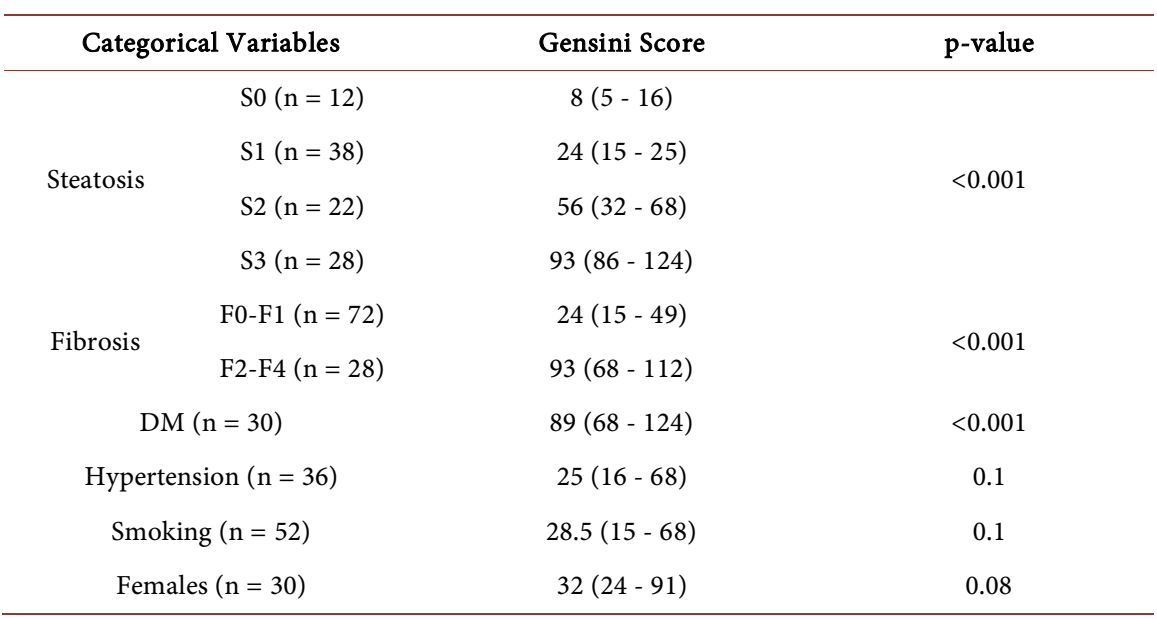

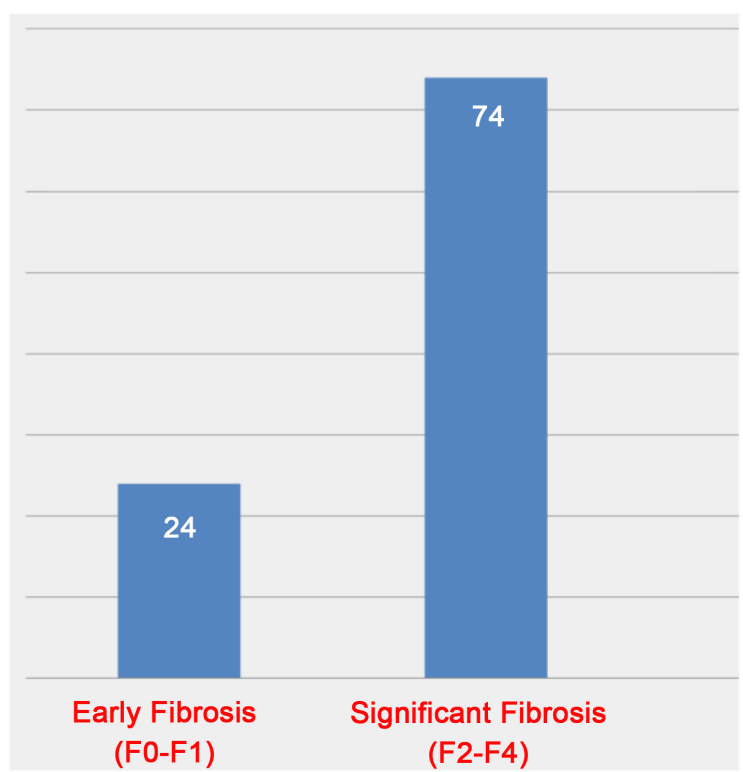

Figure 1. Difference between the median Gensini score in patients with early fibrosis (F0-F1) versus those with significant fibrosis (F2-F4), $\mathrm{p}=0.02$. 


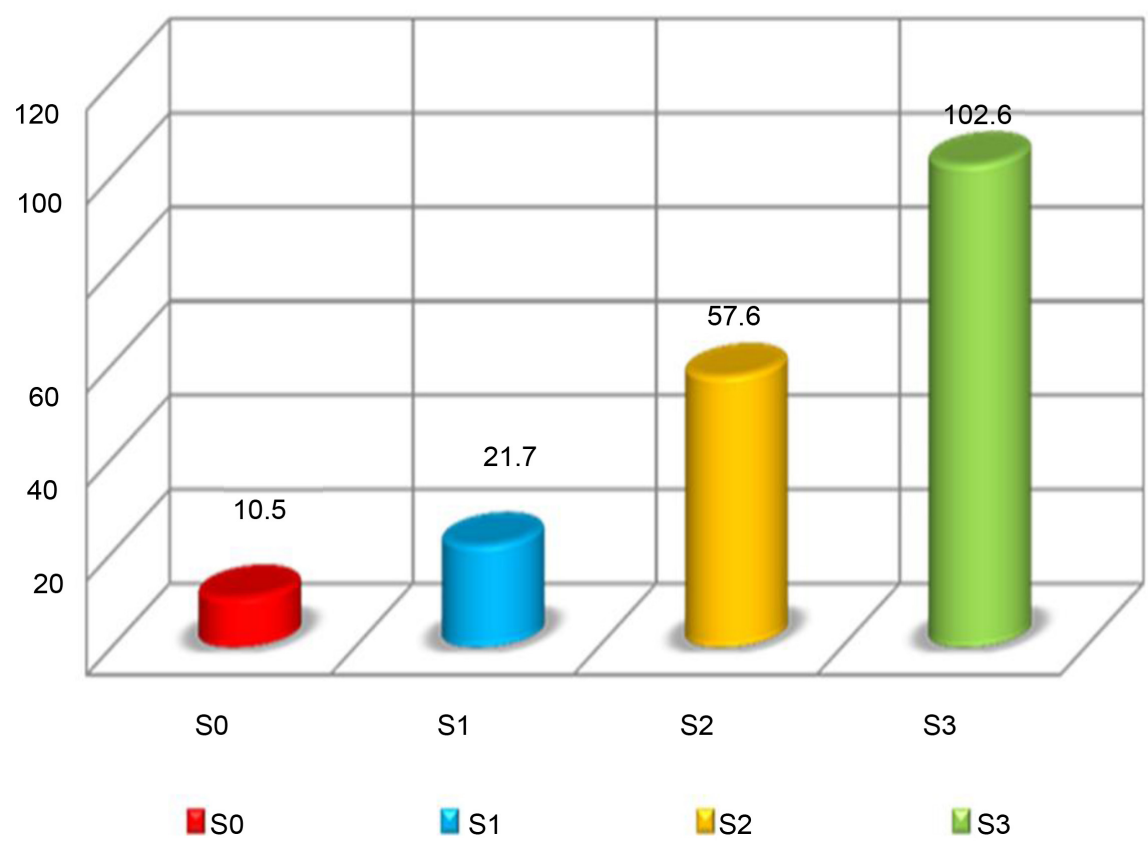

Figure 2. Difference among Gensini scores in patients with the different steatosis groups, $\mathrm{p}=0.01$.

score with the higher steatosis grades; medians: 10.5, 21.7, 57.6 and 102.6 in S0, $\mathrm{S} 1, \mathrm{~S} 2$ and S3 respectively, $\mathrm{p}=0.01$.

Independent variables that have significantly predicted higher Gensini score in univariable regression analysis were: diabetes (OR: 55.6, 95\% CI: $(41-70), \mathrm{p}=$ 0.001), hypertension (OR: 19, 95\% CI: (2 - 37), $\mathrm{p}=0.02), \mathrm{F} 2-\mathrm{F} 4$ fibrosis (OR: 33, 95\% CI: (24 - 38), p = 0.001) and S2-S3 steatosis (OR: 34, 95\% CI: $(23-40), \mathrm{p}=$ $0.001)$, while in multivariable regression analysis; the statistically significant variables were: diabetes (OR: 23, 95\% CI: $(10-37), \mathrm{p}=0.001)$, hypertension (OR: $11,95 \%$ CI: $(4.8-22), \mathrm{p}=0.04)$ and S2-S3 steatosis (OR: 24, 95\% CI: $(17-31), \mathrm{p}=$ $0.001)$, Table 4.

\section{Discussion}

Regarding its pathologic features, disease onset and progression, NAFLD is strongly linked to obesity, diabetes and metabolic syndrome. However; recent studies have recognized NAFLD as a separate entity independent of these diseases based on the notice that not all diabetics have NAFLD and not all NAFLD patients are obese [9] [15], also genetic predisposition to NAFLD has recently been identified in genome-wide studies [16] [17] [18] [19]. Therefore, NAFLD may play a direct role in the development and progression of CVD [20]. In the other hand; as CVD is the most common cause of death in patients with NAFLD, the 2016 European Association for the Study of the Liver (EASL) and the 2018 American Association for the Study of Liver Diseases (AASLD) guidelines recommend mandatory screening for cardiovascular health and aggressive modification of CVD risk for all NAFLD people [9] [15]. 
Table 4. Univariate and multivariate regression of the studied variables.

\begin{tabular}{ccccc}
\hline \multirow{2}{*}{$\begin{array}{c}\text { Independent } \\
\text { Variable }\end{array}$} & Odds Ratio & p-value & Odds Ratio & p-value \\
\cline { 2 - 5 } & $55.6(41-70.5)$ & 0.001 & $23.4(10-37)$ & 0.001 \\
Diabetes & $19.4(2.1-37)$ & 0.02 & $11(4.8-22)$ & 0.04 \\
Hypertension & $16(3-34)$ & 0.08 & $3.8(0.6-4.8)$ & 0.4 \\
Gender & $0.2(0.1-0.3)$ & 0.1 & $0.04(0.01-0.2)$ & 0.5 \\
Cholesterol & $0.07(0.002-0.2)$ & 0.05 & $0.04(0.01-0.09)$ & 0.1 \\
Triglycerides & $1.1(0.4-2.2)$ & 0.05 & $0.5(0.2-1.2)$ & 0.1 \\
HDL & $33(24-38)$ & 0.001 & $7.6(0.7-15.8)$ & 0.07 \\
Fibrosis (F2-F4) & $34(23-40)$ & 0.001 & $24(17-31)$ & 0.001 \\
Steatosis (S2-S3) & & & & \\
\hline
\end{tabular}

In two cross-sectional studies by Targher et al. [21] [22] on 343 type 1 and 2839 type 2 diabetics, authors have concluded that NAFLD is associated with higher prevalence of CVD after adjusting for conventional CVD risk factors and metabolic syndrome components with odds ratios of 7.6 (CI: 3.6 - 24) and 1.49 (CI: 1.1 - 2) respectively. Interestingly; a meta-analysis of 34 studies reported an association between NAFLD and increased risk of prevalent and incident CVD (OR: 1.81, CI: 1.23 - 2.66) and (HR: 1.37, CI: 1.10 - 1.72) respectively, but no association was reported between it and the cardiovascular overall mortality [23].

Our current study yielded similar relationship between S2-S3 NAFLD and CVD (OR: 24, CI: 17 - 31), and despite the smaller number of included patients in our study, the used tools for diagnosis of NAFLD and CVD; either transient elastography and coronary angiography, are more accurate and objective than those in the previous two studies [21] [22] that were observational and depended only on the history for diagnosis of CVD (coronary, cerebrovascular, and peripheral vascular disease), and abdominal US for diagnosis of NAFLD. Figure 3 shows an example of 33-year-old male patient with S3 liver steatosis (CAP = 368) and his angiography findings showed significant stenosis at the left circumflex coronary artery (LCX), chronic total occlusion at the left anterior descending (LAD) and normal right coronary artery (RCA), with moderate-severity coronary ischemia as indicated by Gensini score of 56. Amazingly; this patient had a body mass index of $31 \mathrm{~kg} / \mathrm{m}^{2}$ with no other cardiovascular risk factors.

In contrary to the above-mentioned data, NAFLD as estimated by fatty liver index was not a significant predictor of acute myocardial infarction in a long-term prospective study which also emphasized interplay of confounders [24]. Another large-scale prospective study by Chang et al. has shown similar findings with insignificant association between NAFLD and CVD hospitalization after further adjusting for potential mediators [25].

The discrepancy between the results of the latter two studies and the previous ones could be assumed to the wide array of cardiovascular presentations that considered in each study as well as the variable methods of NAFLD diagnosis which included the US, computerized tomography and non-invasive markers. 

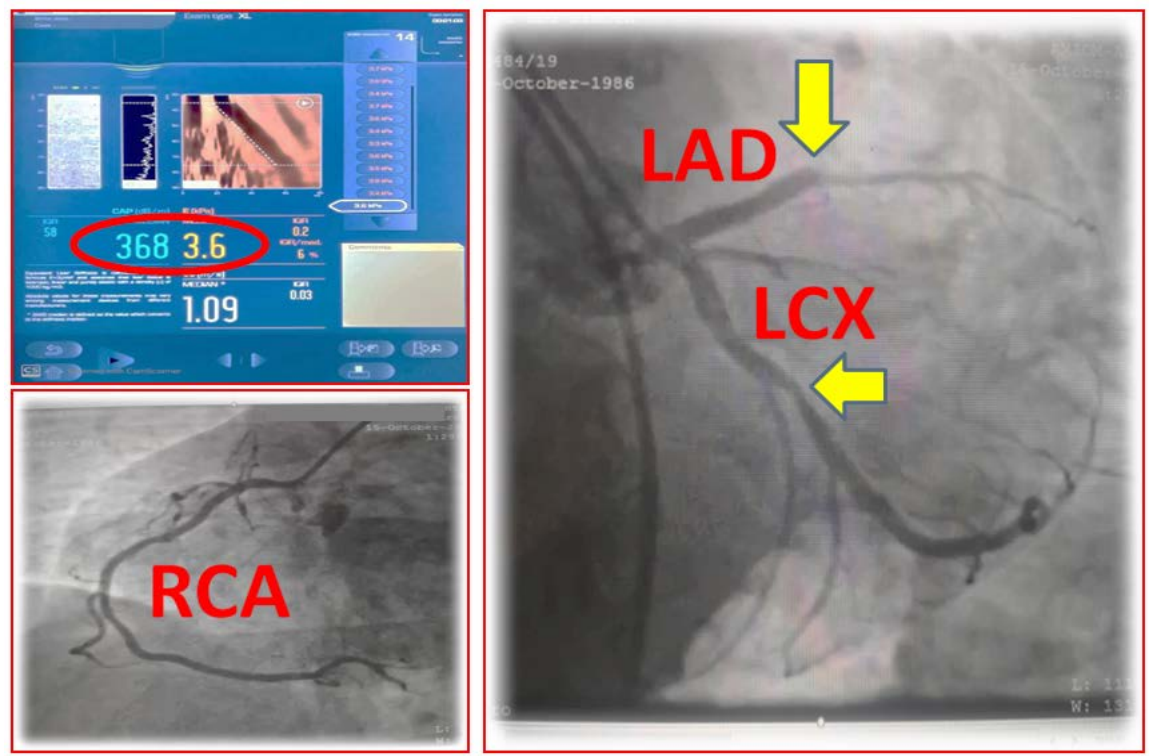

Figure 3. Transient elastography of 33-year-old male patient with S3 liver steatosis (CAP = $368 \mathrm{~dB} / \mathrm{m}$ ), F0-F1 fibrosis (3.6 kilopascal); both appear red-encircled, and angiographic findings showed significant stenosis (yellow horizontal arrow) at the left circumflex coronary artery (LCX), chronic total occlusion (yellow vertical arrow) at the left anterior descending (LAD) and normal right coronary artery (RCA), with calculated Gensini score of 56 .

The main advantage of our study is using CAP as a diagnostic tool for NAFLD which allows not just diagnosis of NAFLD but also its stratification from S0 to S3, and coronary angiography with Gensini score calculation which reflects more realistic assessment of the cardiovascular risk. However, we had certain limitations including small sample size and lack of long-term follow up.

\section{Conclusion}

In conclusion, NAFLD is an independent cardiovascular risk predictor with statistically significant increase in Gensini score with the higher grades of NAFLD.

\section{Acknowledgements}

We acknowledge Dr: Albair Abdul-Maseh for his kind effort in arranging the patients' schedules.

\section{Conflicts of Interest}

The authors declare no conflicts of interest regarding the publication of this paper.

\section{References}

[1] Younossi, Z.M., Stepanova, M., Afendy, M., Fang, Y., Younossi, Y., Mir, H., et al. (2011) Changes in the Prevalence of the Most Common Causes of Chronic Liver Diseases in the United States from 1988 to 2008. Clinical Gastroenterology and Hepatology, 9, 524-530.e521. https://doi.org/10.1016/j.cgh.2011.03.020 
[2] Younossi, Z.M., Koenig, A.B., Abdelatif, D., Fazel, Y., Henry, L. and Wymer, M. (2016) Global Epidemiology of Nonalcoholic Fatty Liver Disease Meta-Analytic Assessment of Prevalence, Incidence, and Outcomes. Hepatology, 64, 73-84. https://doi.org/10.1002/hep.28431

[3] Charlton, M.R., Burns, J.M., Pedersen, R.A., Watt, K.D., Heimbach, J.K. and Dierkhising, R.A. (2011) Frequency and Outcomes of Liver Transplantation for Nonalcoholic Steatohepatitis in the United States. Gastroenterology, 141, 1249-1253. https://doi.org/10.1053/j.gastro.2011.06.061

[4] Targher, G., Day, C.P. and Bonora, E. (2010) Risk of Cardiovascular Disease in Patients with Nonalcoholic Fatty Liver Disease. The New England Journal of Medicine, 363, 1341-1350. https://doi.org/10.1056/NEJMra0912063

[5] Soderberg, C., Stal, P., Askling, J., Glaumann, H., Lindberg, G., Marmur, J., et al. (2010) Decreased Survival of Subjects with Elevated Liver Function Tests during a 28-Year Follow-Up. Hepatology, 51, 595-602. https://doi.org/10.1002/hep.23314

[6] Ekstedt, M., Hagstrom, H., Nasr, P., Fredrikson, M., Stal, P., Kechagias, S., et al. (2015) Fibrosis Stage Is the Strongest Predictor for Disease-Specific Mortality in NAFLD after up to 33 Years of Follow-Up. Hepatology, 61, 1547-1554. https://doi.org/10.1002/hep.27368

[7] Emrich, I.E., Böhm, M. and Mahfoud, F. (2019) The 2018 ESC/ESH Guidelines for the Management of Arterial Hypertension: A German Point of View. European Heart Journal, 40, 1830-1831. https://doi.org/10.1093/eurheartj/ehz381

[8] Arnett, D.K., Khera, A. and Blumenthal, R.S. (2019) ACC/AHA Guideline on the Primary Prevention of Cardiovascular Disease: Part 1, Lifestyle and Behavioral Factors. JAMA Cardiology, 4, 1043-1044. https://doi.org/10.1001/jamacardio.2019.2604

[9] Chalasani, N., Younossi, Z., Lavine, J.E., Charlton, M., Cusi, K., Rinella, M., Harrison, S.A., Brunt, E.M. and Sanyal, A.J. (2018) The Diagnosis and Management of Nonalcoholic Fatty Liver Disease: Practice Guidance from the American Association for the Study of Liver Diseases. Hepatology, 67, 328-357. https://doi.org/10.1002/hep.29367

[10] Tag-Adeen, M., Sabra, A.M., Akazawa, Y., Ohnita, K. and Nakao, K. (2017) Impact of Hepatitis C Virus Genotype-4 Eradication Following Direct Acting Antivirals Treatment on Liver Stiffness Measurement. Hepatic Medicine, Evidence and Research, 9, 45-53. https://doi.org/10.2147/HMER.S142600

[11] Castera, L., Vergniol, J., Foucher, J., et al. (2005) Prospective Comparison of Transient Elastography, Fibrotest, APRI, and Liver Biopsy for the Assessment of Fibrosis in Chronic Hepatitis C. Gastroenterology, 128, 343-350.

https://doi.org/10.1053/j.gastro.2004.11.018

[12] Sasso, M., Miette, V., Sandrin, L. and Beaugrand, M. (2012) The Controlled Attenuation Parameter (CAP): A Novel Tool for the Non-Invasive Evaluation of Steatosis Using Fibroscan. Clinics and Research in Hepatology and Gastroenterology, 36, 13-20. https://doi.org/10.1016/j.clinre.2011.08.001

[13] Neeland, I.J., Patel, R.S., Eshtehardi, P., Dhawan, S., McDaniel, M.C., Rab, S.T., et al. (2012) Coronary Angiographic Scoring Systems: An Evaluation of Their Equivalence and Validity. American Heart Journal, 164, 547-552e1. https://doi.org/10.1016/j.ahj.2012.07.007

[14] Gensini, G.G. (1983) A More Meaningful Scoring System for Determining the Severity of Coronary Heart Disease. American Journal of Cardiology, 51, 606. https://doi.org/10.1016/S0002-9149(83)80105-2

[15] European Association for the Study of the Liver, European Association for the 
Study of Diabetes and European Association for the Study of Obesity (2016) EASL-EASD-EASO Clinical Practice Guidelines for the Management of Nonalcoholic Fatty Liver Disease. Journal of Hepatology, 64, 1388-1402.

[16] Liu, Y.L., Reeves, H.L., Burt, A.D., Tiniakos, D., McPherson, S., Leathart, J.B., et al. (2014) TM6SF2 rs58542926 Influence Shepatic Fibrosis Progression in Patients with Non-Alcoholic Fatty Liver Disease. Nature Communications, 5, 4309. https://doi.org/10.1038/ncomms5309

[17] Dongiovanni, P., Petta, S., Maglio, C., Fracanzani, A.L., Pipitone, R., Mozzi, E., et al. (2015) Transmembrane 6 Superfamilymember 2 Gene Variant Disentangles Nonalcoholic Steatohepatitis from Cardiovascular Disease. Hepatology, 61, 506-514. https://doi.org/10.1002/hep.27490

[18] Valenti, L., Alisi, A., Galmozzi, E., Bartuli, A., Del Menico, B., Alterio, A., et al. (2010) I148M Patatin-Like Phospholipasedomain-Containing 3 Gene Variant and Severity of Pediatric Nonalcoholic Fatty Liver Disease. Hepatology, 52, 1274-1280. https://doi.org/10.1002/hep.23823

[19] Nobili, V., Donati, B., Panera, N., Vongsakulyanon, A., Alisi, A., Dallapiccola, B., et al. (2014) A 4-Polymorphism Risk Scorepredicts Steatohepatitis in Children with Nonalcoholic Fatty Liver Disease. The Journal of Pediatric Gastroenterology and Nutrition, 58, 632-636. https://doi.org/10.1097/MPG.0000000000000279

[20] Zhou, Y.Y., Zhou, X.D., Wu, S.J., Hu, X.Q., Tang, B., Poucke, S.V., Pan, X.Y., Wu, W.J., Gu, X.M., Fu, S.W., et al. (2018) Synergistic Increase in Cardiovascular Risk in Diabetes Mellitus with Nonalcoholic Fatty Liver Disease: A Meta-Analysis. European Journal of Gastroenterology \& Hepatology, 30, 631-636. https://doi.org/10.1097/MEG.0000000000001075

[21] Targher, G., Pichiri, I., Zoppini, G., Trombetta, M. and Bonora, E. (2012) Increased Prevalence of Cardiovascular Disease in Type 1 Diabetic Patients with Non-Alcoholic Fatty Liver Disease. Journal of Endocrinological Investigation, 35, 535-540. https://doi.org/10.1111/j.1464-5491.2011.03427.x

[22] Targher, G. and Arcaro, G. (2007) Non-Alcoholic Fatty Liver Disease and Increased Risk of Cardiovascular Disease. Atherosclerosis, 191, 235-240. https://doi.org/10.1016/j.atherosclerosis.2006.08.021

[23] Wu, S., Wu, F., Ding, Y., Hou, J., Bi, J. and Zhang, Z. (2016) Association of Non-Alcoholic Fatty Liver Disease with Major Adverse Cardiovascular Events: A Systematic Review and Meta-Analysis. Scientific Reports, 6, Article No. 33386. https://doi.org/10.1038/srep33386

[24] Olubamwo, O.O., Virtanen, J.K., Voutilainen, A., Kauhanen, J., Pihlajamäkia, J. and Tuomainen, T.-P. (2018) Association of Fatty Liver Index with the Risk of Incident Cardiovascular Disease and Acute Myocardial Infarction. European Journal of Gastroenterology \& Hepatology, 30, 1047-1054. https://doi.org/10.1097/MEG.0000000000001183

[25] Chang, Y., Ryu, S., Sung, K.C., Cho, Y.K., Sung, E., Kim, H.N., Jung, H.S., Yun, K.E., Ahn, J., Shin, H., et al. (2019) Alcoholic and Non-Alcoholic Fatty Liver Disease and Associations with Coronary Artery Calcification: Evidence from the Kangbuk Samsung Health Study. Gut, 68, 1667-1675. https://doi.org/10.1136/gutjnl-2018-317666 


\section{Abbreviations}

CAP: Controlled Attenuation Parameter.

CLD: Chronic Liver Disease.

CVD: Cardiovascular Disease.

LSM: Liver Stiffness Measurement.

NAFLD: Non-Alcoholic Fatty Liver Disease.

NASH: Non-Alcoholic Steatohepatitis.

TE: Transient Elastography.

US: Abdominal Ultrasound. 